\title{
Fine Motor Delay
}

National Cancer Institute

\section{Source}

National Cancer Institute. Fine Motor Delay. NCI Thesaurus. Code C116602.

Delayed acquisition of age appropriate motor milestones that produce small and precise movements. 